\title{
Genome assembly of Danaus chrysippus and comparison with the Monarch Danaus plexippus
}

\author{
Kumar Saurabh Singh (D) , ${ }^{1, \dagger}$ Rishi De-Kayne (D) , ${ }^{2,+}$ Kennedy Saitoti Omufwoko (D) , ${ }^{3}$ Dino J. Martins (D) ${ }^{3,4}$ Chris Bass (D) ${ }^{5}$ \\ Richard ffrench-Constant (D), ${ }^{5}$ Simon H. Martin (DD ${ }^{2, \star}$ \\ ${ }^{1}$ Bioinformatics Group, Wageningen University, Wageningen 6708 PB, The Netherlands, \\ ${ }^{2}$ Institute of Evolutionary Biology, University of Edinburgh, Edinburgh EH9 3FL, UK, \\ ${ }^{3}$ Department of Ecology and Evolutionary Biology, Princeton University, Princeton, NJ 08544, USA, \\ ${ }^{4}$ Mpala Research Centre, Nanyuki, P O Box 555 10400, Kenya, \\ ${ }^{5}$ Centre for Ecology and Conservation, University of Exeter, Penryn TR10 9FE, UK \\ *Corresponding author: Institute of Evolutionary Biology, University of Edinburgh, Edinburgh EH16 6DX, UK. Email: simon.martin@ed.ac.uk \\ ${ }^{\dagger}$ These authors contributed equally to this study.
}

\begin{abstract}
Milkweed butterflies in the genus Danaus are studied in a diverse range of research fields including the neurobiology of migration, biochemistry of plant detoxification, host-parasite interactions, evolution of sex chromosomes, and speciation. We have assembled a nearly chromosomal genome for Danaus chrysippus (known as the African Monarch, African Queen, and Plain Tiger) using long-read sequencing data. This species is of particular interest for the study of genome structural change and its consequences for evolution. Comparison with the genome of the North American Monarch Danaus plexippus reveals generally strong synteny but highlights 3 inversion differences. The 3 chromosomes involved were previously found to carry peaks of intraspecific differentiation in D. chrysippus in Africa, suggesting that these inversions may be polymorphic and associated with local adaptation. The $D$. chrysippus genome is over $40 \%$ larger than that of $D$. plexippus, and nearly all of the additional $\sim 100$ Megabases of DNA comprises repeats. Future comparative genomic studies within this genus will shed light on the evolution of genome architecture.
\end{abstract}

Keywords: African Monarch; African Queen; Plain Tiger; repeat content; intron length; genome size; butterfly

\section{Introduction}

The genus Danaus is perhaps best known for the iconic Monarch butterfly Danaus plexippus and its extraordinary migrations in North America. Genomic studies of the Monarch have shed light on host plant detoxification (Tan et al. 2019), sex chromosome evolution (Mongue et al. 2017; Gu et al. 2019), and the genetic basis of migratory behavior (Zhan et al. 2014). Its relative Danaus chrysippus is found throughout Africa, the Mediterranean, and south Asia, and is known as the African Monarch, African Queen, and Plain Tiger butterfly in different parts of its range. Danaus chrysippus is emerging as a useful study system in evolutionary genomics. Several subspecies of D. chrysippus with distinct warning patterns occupy distinct geographic ranges separated by broad hybrid zones (Smith et al. 1997; Lushai et al. 2003). Patterns of genetic differentiation suggest a role for chromosomal rearrangements in maintaining these differences (Martin et al. 2020). In the east African hybrid zone, a neo-W sex chromosome has emerged in the past few thousand years and is associated with infection by a male-killing endosymbiont Spiroplasma (Smith et al. 2016; Martin et al. 2020). This species therefore has great potential for future research on the evolutionary impacts of genome structural change.
Here, we describe the generation of a chromosome-level assembly for D. chrysippus based on Pacific Biosciences long-read sequencing data. This serves to replace a previous reference genome based on short-read sequences and mate-pair libraries, which had low contiguity ( $\mathrm{N} 50=0.63 \mathrm{Mb}$, Martin et al. 2020). Our new assembly has an N50 of $11.45 \mathrm{Mb}$. Nineteen of the 30 chromosomes are represented by a single contig and the remaining 11 by 2 contigs each. At $354 \mathrm{Mb}$, this genome is average in size for a butterfly, but about 1.4 times larger than that of $D$. plexippus ( $250 \mathrm{Mb})$. Comparative analyses indicate that this difference is largely explained by increased repeat content, but D. chrysippus also has larger introns, implying that these species have experienced different selection pressures acting on nonessential DNA.

\section{Materials and methods DNA sequencing}

High-molecular-weight DNA was extracted from a single female pupa from a captive butterfly stock using the Qiagen Blood \& Cell Culture DNA Mini Kit following the manufacturer's guidelines. Long-read Pacific Biosciences sequencing was performed using 7 PacBio Sequel SMRT cells on the Sequel platform, yielding 
approximately 9.7 gigabases $(\mathrm{Gb})$ per SMRT cell. The 3.8 million PacBio reads totaled $67.6 \mathrm{~Gb}$ and had an N50 of $27.3 \mathrm{~kb}$. In addition, we generated Illumina sequencing data for the same individual on the Novaseq 600 platform (118 million paired-end reads of $150 \mathrm{bp}$ with an insert size of $350 \mathrm{bp}$ ) totaling $35 \mathrm{~Gb}$.

\section{Genome assembly}

We assembled the long reads using both Canu (Koren et al. 2017) and Falcon (Chin et al. 2016) and then merged these assemblies to maximize the genome completeness using quickmerge - $\mathrm{v} 0.3$ (Chakraborty et al. 2016). Redundant contigs or haplotigs were removed using Purge_haplotigs -v 1.0.4 (Roach et al. 2018) with the -align_cou (Percent cutoff for identifying a contig as haplotig) value of 65 . Before merging, assemblies were polished iteratively using 3 rounds of Pilon -v 1.22 in diploid mode (Walker et al. 2014; using a trimmed version of the short-read data; reads were trimmed using Trim_Galore -v 0.4.0; Krueger 2012), and Racon -V 1.3.1 (Vaser et al. 2017; using the long-read data). Illumina and PacBio raw reads are archived under European Nucleotide Archive project accession: PRJEB47812.

\section{Whole-genome alignment and synteny assessment}

To assess synteny and putatively assign contigs to chromosomes, we aligned the D. chrysippus assembly to 2 D. plexippus assemblies: "Dplex_v4," a chromosome-level assembly produced by scaffolding 4,115 scaffolds using chromatin conformation (Hi-C) data (GCA_009731565.1; Gu et al. 2019) and "MEX_DaPlex," a long-read based assembly consisting of 66 scaffolds, of which 38 (97\% of total sequence) have been assigned to chromosomes (GCA_018135715.1; Ranz et al. 2021). Alignments were generated using both MUMmer's nucmer tool version 3.1 (Marçais et al. 2018), with default parameters except the "maxGap" parameter set to 1,000, and with minimap2 v2.17 (Li 2018), using the "asm20" parameter preset, designed for whole-genome alignment of species with sequence divergence below 20\%. Nucmer alignments were explored using the interactive alignment visualization tool Dot (https:// github.com/dnanexus/dot) and final alignment plots based on minimap2 alignments were generated using Asynt (https://github. com/simonhmartin/asynt version 0.1).

\section{Correcting putative misassemblies}

Visualization of whole-genome alignment to both D. plexippus assemblies (described above) revealed 2 putatively misassembled contigs that had portions aligning confidently to 2 different chromosomes. Although these could theoretically represent real translocation or fusion products, we took the conservative decision to split these contigs, as additional long-read assemblies generated in a related study (Kim et al. 2021) showed no evidence for translocations or fusions. Optimal split points were identified by visual inspection of the alignments, as well as additional BLASTn alignments made between the 2 genomes. The original unsplit assembly, along with details of split points, is available at https://doi.org/10.5281/zenodo.5731560.

Despite having performed automated removal of redundant contigs using Purge_haplotigs, visual exploration of the alignments identified a further 4 contigs that appeared to be redundant (i.e. representing a part of the genome already represented by a larger contig). These included one of the split products described above. To confirm this, we aligned the Illumina reads for the assembled individual back to the assembly using BWA MEM (Li and Durbin 2010) using default parameters, and computed read depth using Samtools depth (Li et al. 2009). Visualization of median read depth averaged in 50-kb windows confirmed that these 4 contigs had $50 \%$ depth, so they were removed from the assembly. Finally, 2 contigs included portions that appeared to be redundant in the alignments as well as read depth plots. These were therefore split at the point in the alignment where the redundancy began, and the redundant fragment was removed from the final assembly. The full original assembly, along with details of all splits and portions retained to produce the final Dchry2.2 assembly, is available at https://doi.org/10.5281/zen odo.5731560. To assess the base-level accuracy of our assembly, we calculated the consensus quality $(\mathrm{QV})$, comparing the frequency of $\mathrm{k}$-mers present in the raw Illumina reads with those present across the final assembly (all 83 contigs) using Merqury v.1.3 (Rhie et al. 2020).

\section{Repeat annotation}

To assess the repeat content of the assembly, the genome was masked using a custom repeat library. First, a repeat library was produced using the finished genome assembly, using RepeatModeler v2.0.1 (Smit and Hubley 2008), and this library was then combined with a broad Lepidoptera repeat database extracted using RepeatMasker v.4.1.0 (Smit et al. 2015). Repeat masking of the genome was then carried out using RepeatMasker (Smit et al. 2015). To determine the prevalence of expanding transposable element families within D. chrysippus, the scripts calDivergenceFromAlign.pl and createRepeatLandscape.pl from RepeatModeler (Smit and Hubley 2008) were used to produce a repeat landscape for the assembly. To facilitate a comparison with other Danaus genome assemblies, this repeat masking process was repeated using the same custom repeat library for 2 well-assembled D. plexippus genome assemblies [NCBI accessions GCF_009731565.1 and GCA_018135715.1 (Ranz et al. 2021)]. The resulting soft masked assemblies were then used for genome annotation.

\section{Gene annotation}

Due to a lack of RNAseq data, a preliminary genome annotation was carried out using 2 protein sets from the close relative to D. chrysippus, D. plexippus, the Monarch butterfly. This combined protein set was produced by collating protein information from 2 different, D. plexippus assemblies, the first a proteome downloaded from uniprot under the accession UP000596680 (associated with the Dplex_v4 assembly), and the second taken by extracting amino acid sequences from the annotation of the "MEX_DaPlex" D. plexippus assembly GCF_009731565.1 (Ranz et al. 2021). Both protein sets had high Benchmarking Universal Single-Copy Orthologs (BUSCO) scores, indicative of high-quality annotation (Simão et al. 2015). This combined protein set was then used as input for the BRAKER2 pipeline (Brina et al. 2021) to annotate each of the 3 soft masked genome assemblies produced above (specifying -gff3 -softmasking -prot_seq=protein_set.fasta prg=gth -gth2traingenes -trainFromGth). GenomeTools (Gremme et al. 2013) was then used to sort and tidy the annotation output (gt gff -sort -tidy -retainids -fixregionboundaries) and calculate summary statistics of the annotation (gt stat -genelengthdistri -genescoredistri -exonlengthdistri exonnumberdistri -intronlengthdistri -cdslengthdistri). Functional annotation for the resulting D. chrysippus protein set was carried out using Pannzer2 (Törönen et al. 2018). To determine variation in intron and exon length between 
(a)
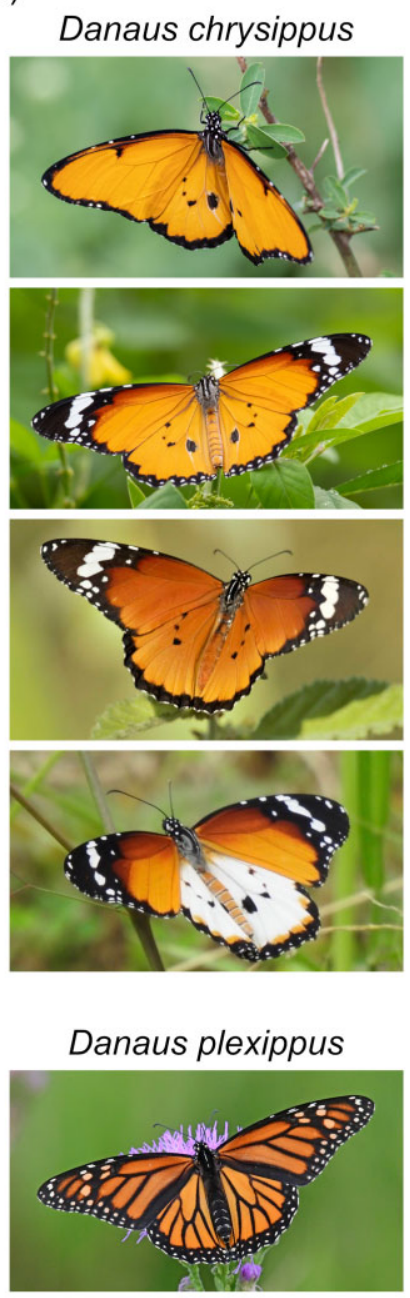

(b)

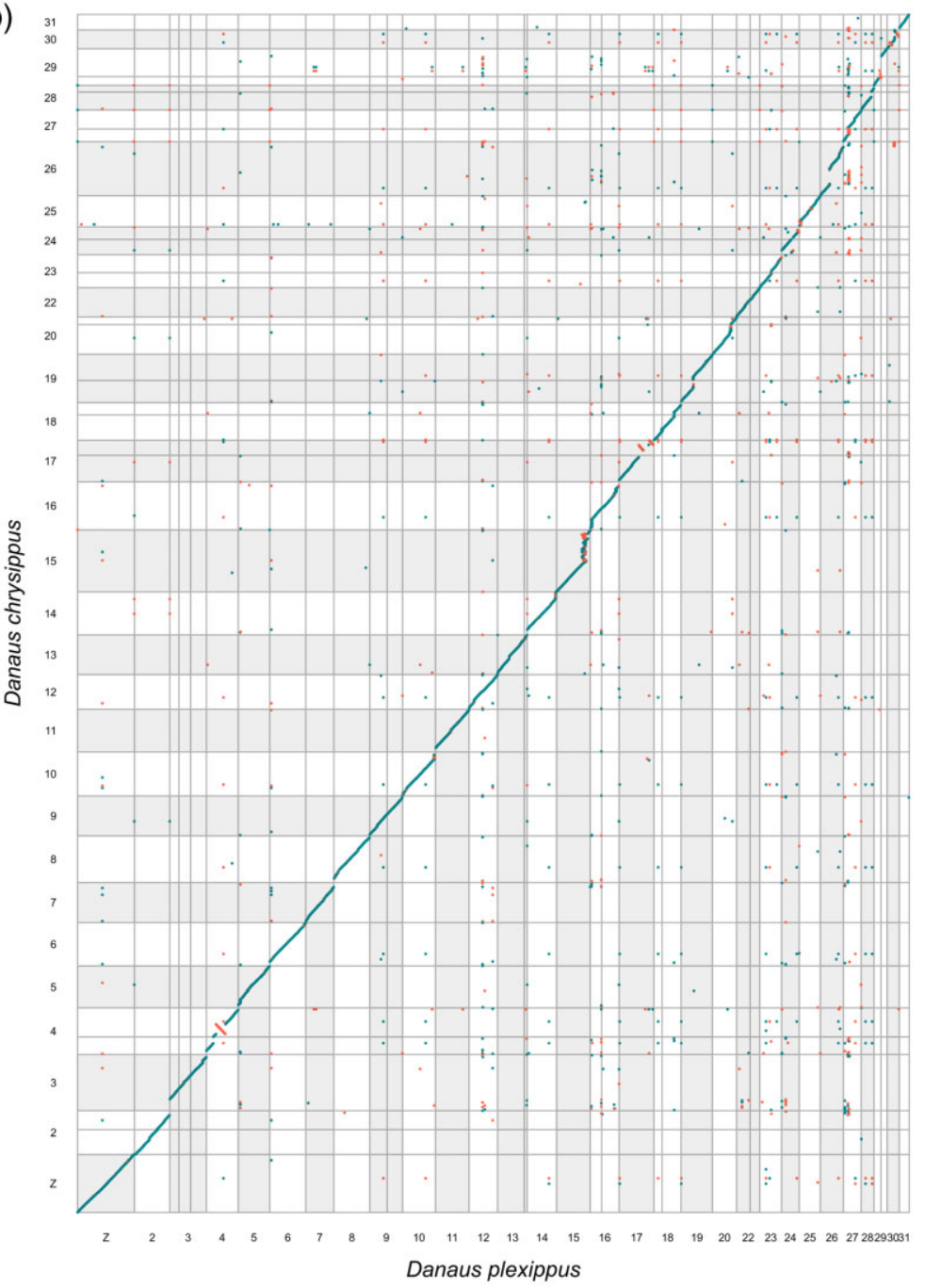

Fig. 1. a) Four color morphs of D. chrysippus (above) and D. plexippus (below). b) Whole-genome alignment between D. chrysippus and D. plexippus (MEX_DaPlex assembly). Points represent minimap2 alignments greater than $5 \mathrm{~kb}$ in length. Alignments in the same orientation are shown in turquoise and those in the reverse orientation are shown in red. Only contigs that were confidently assigned to chromosomes $(97 \%$ of the total in both assemblies) are included. Alternating grey and white bars indicate separate chromosomes. See Supplementary Fig. 1 for the same plot including contig/ scaffold labels. Butterfly images from top to bottom by Forest Jarvis (CC-BY-NC), Paul Dickson (CC-BY-NC), Claude Martin, Steven Schulting (CC-BYNC), and Edward Perry IV (CC-BY-NC).

D. chrysippus and D. plexippus, introns and exons were extracted from our corresponding annotation file for each of the 3 assemblies.

\section{Genome comparison and assembly validation}

To assess the quality of annotation, BUSCO scores were calculated for both the full D. chrysippus assembly and the protein sequences resulting from annotation specifying the insecta_odb10 lineage BUSCO set in BUSCO v.5.0.0 (Simão et al. 2015). Additionally, the annotation was compared against those of both published D. plexippus annotations. To compare each of the assemblies, and in turn, the consistency of genome structure across Danaus species we plotted the distribution of intron lengths for annotations from each of the 3 assemblies. This was carried out by extracting introns and exons from the longest annotated transcript for each gene within each of the annotations (using the BRAKER2 re-annotations for both D. plexippus assemblies to ensure lengths were comparable across assemblies).

\section{Results and Discussion Genome assembly}

In total, $67.6 \mathrm{~Gb}$ of long-read data was assembled into 83 contigs. Manual splitting of 3 putatively misassembled contigs and removal of several remaining redundant fragments (see Materials and Methods) left 83 contigs with an N50 of $11.45 \mathrm{Mb}$ and L50 of 13 contigs, giving a total genome size of $354 \mathrm{Mb}$. Alignment with 2 different $D$. plexippus assemblies allowed us to confidently assign 41 contigs representing $97 \%$ of the sequence length to chromosomes (Fig. 1). Of the 30 D. chrysippus chromosomes, 19 are represented by a single contig and the rest by 2 contigs each. The contiguity of our assembly is therefore comparable to that of the D. plexippus "MEX_DaPlex" assembly (Ranz et al. 2021), for which 38 out of 66 scaffolds ( $97 \%$ of the assembly) were assigned to chromosomes, of which 23 are represented by a single scaffold. Among the $42 \mathrm{D}$. chrysippus contigs that were not assigned to a chromosome ( $3 \%$ of the genome), it is possible that some represent fragments of the female-specific W chromosome. However, given that butterfly $\mathrm{W}$ chromosomes are highly 


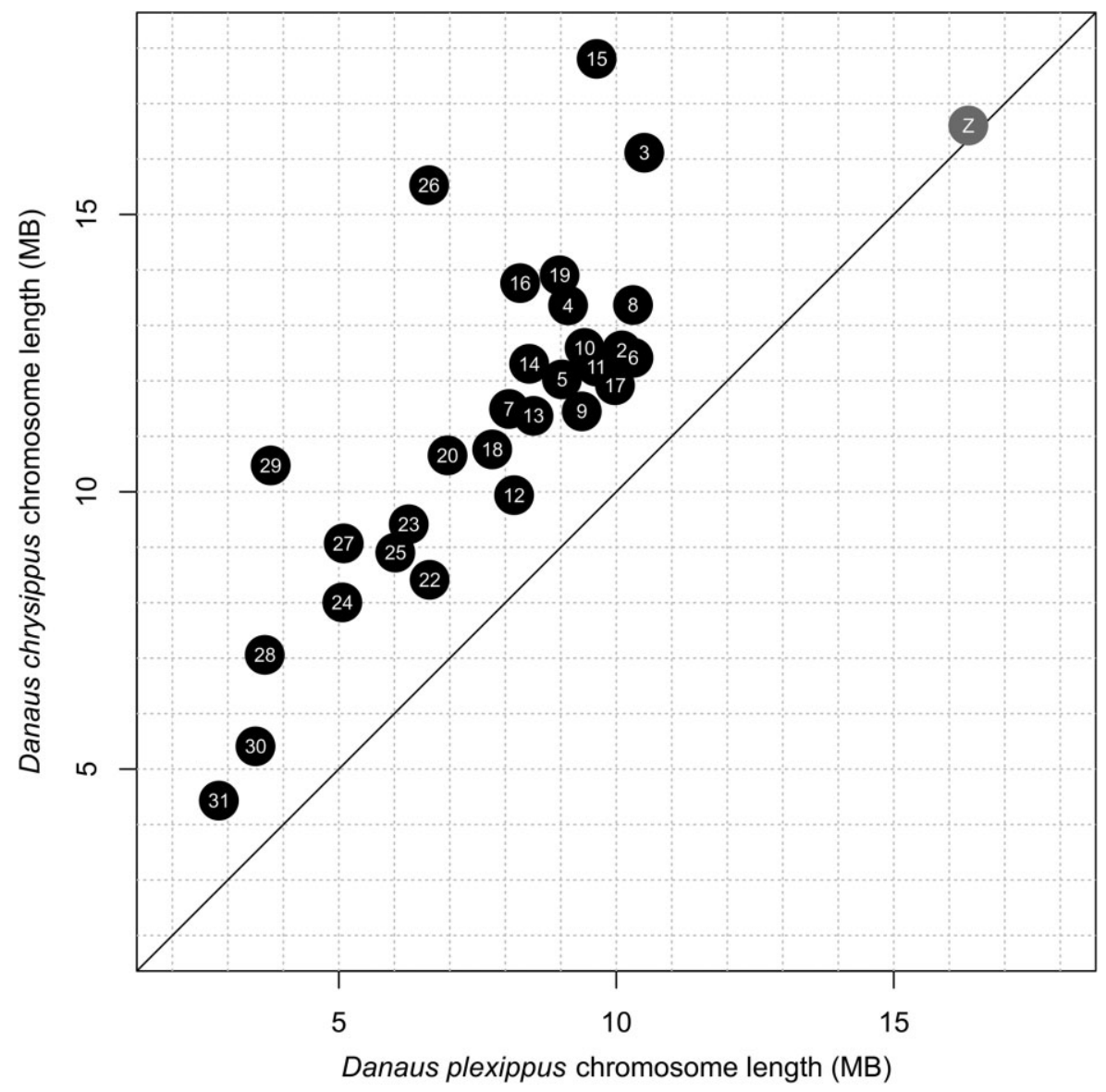

Fig. 2. Chromosome length comparison between D. chrysippus and D. plexippus (MEX_DaPlex assembly). Chromosome lengths represent the sum total of the contigs/scaffolds assigned to each chromosome. Autosomes are shown in black and the Z sex chromosome in grey.

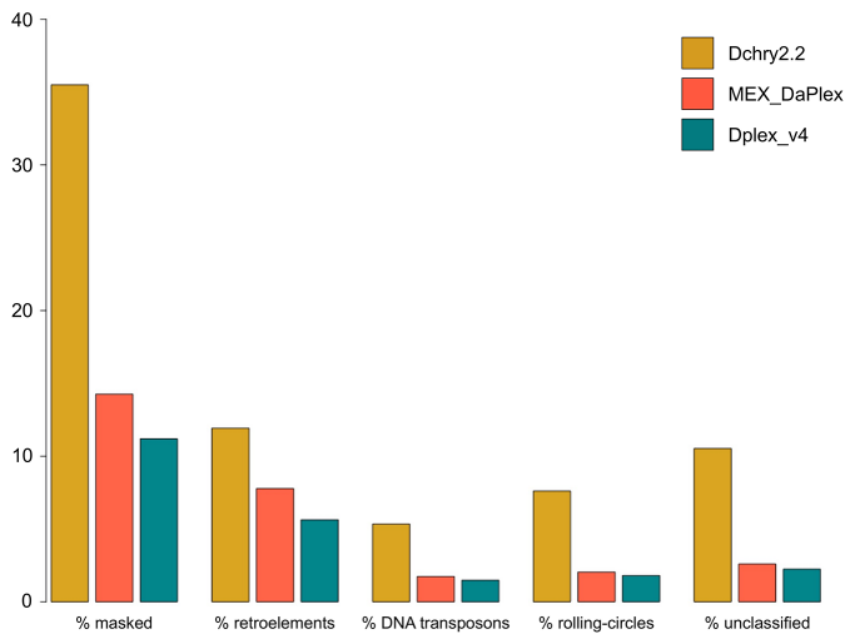

Fig. 3. Barplot showing the proportion of the genome of Danaus chrysippus (yellow) and D. plexippus (represented by both the MEX_DaPlex, in red, and Dplex_v4, in turquoise, assemblies) made up of repetitive elements (as identified and masked by repeatmodeler and repeatmasker). Additionally, the proportion of each genome made up of specific repetitive element families is shown highlighting the increased proportion of repetitive elements in Danaus chrysippus.

repetitive and have low interspecific homology (Lewis et al. 2021), further work comparing male and female genomes is required to test this hypothesis. The genome-wide consensus quality of the assembly (QV; representing a log-scaled probability of error for each base in our assembly) was 36.2373, suggesting a high level of accuracy (equating to an accuracy between 99.9\% and $99.99 \%$ ).

\section{Synteny and genome size comparison}

The genomes of $D$. chrysippus and D. plexippus are largely syntenic (Fig. 1). Our assembly supports the earlier finding that the $Z$ sex chromosome of Danaus species represents a fusion between the ancestral lepidopteran Z chromosome and autosome 21, which occurred in a recent ancestor of the genus (Mongue et al. 2017). We numbered chromosomes according to their homologs in the most recent D. Plexippus assembly (Ranz et al. 2021), which follows the growing convention of using the chromosome numbering system introduced for Melitaea cinxia, the first assembled lepidopteran genome that retains the ancestral karyotype of 31 (Ahola et al. 2014; Cicconardi et al. 2021; Lewis et al. 2021; Ranz et al. 2021). As such, the Danaus karyotype lacks an autosome 21, as this is now part of the $\mathrm{Z}$ sex chromosome.

Several putative inversion differences can be identified between the 2 Danaus species, most notably on chromosomes 4, 17, and 30 (Fig. 1). We note that all 3 of these chromosomes were found to carry sharp peaks of intraspecific differentiation between subspecies of D. chrysippus in Africa, against a background of very low genetic differentiation (Martin et al. 2020), suggesting that these putative inversions may be polymorphic and associated with local adaptation in D. chrysippus. In addition, chromosomes 15, 26, and 29 all carry large duplicated/repetitive portions 


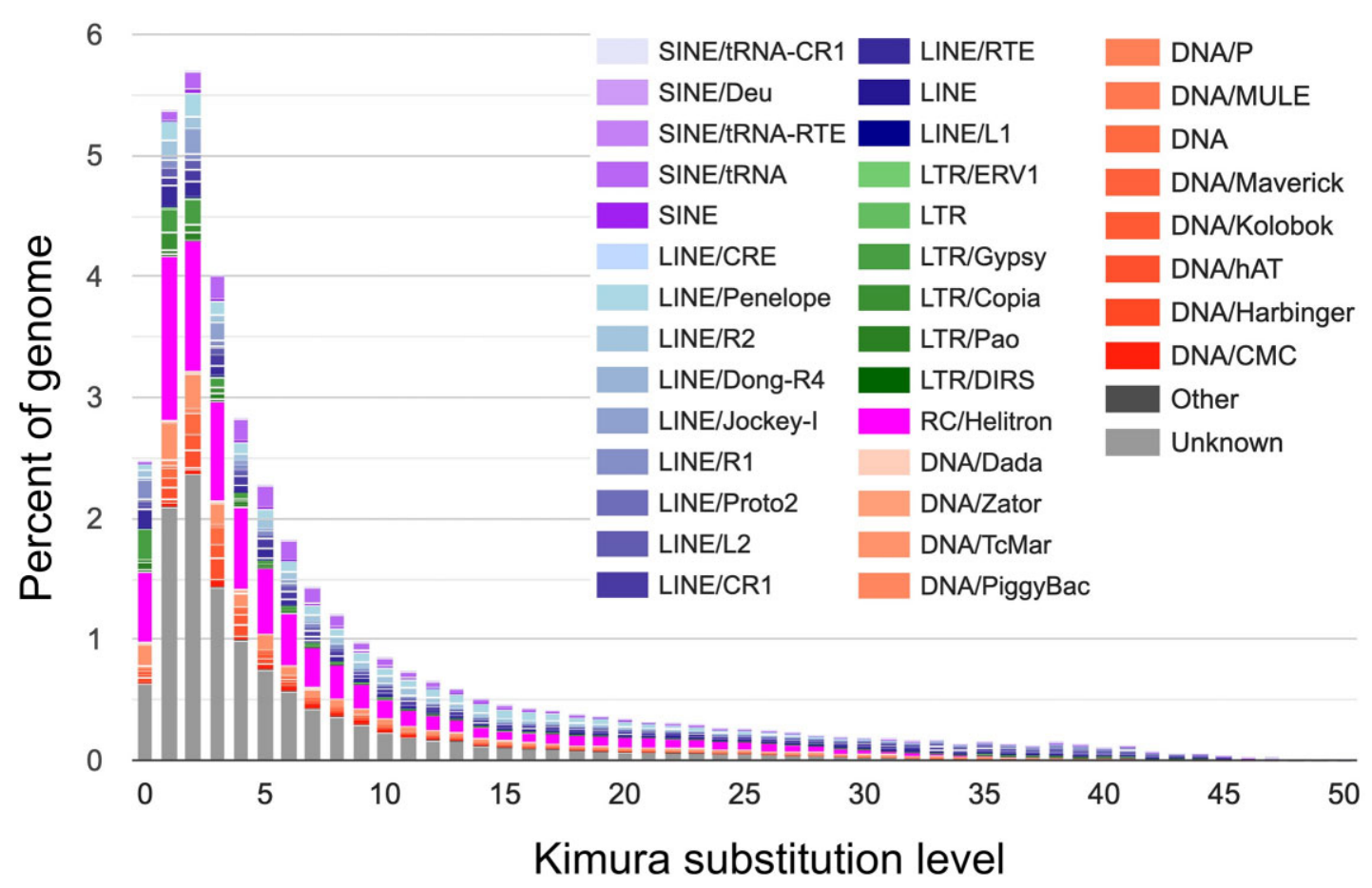

Fig. 4. The repeat landscape of the Dchry2.2 assembly. In addition to unclassified repeats, rolling-circle (RC/Helitron), LINE and LTR families all appear to have expanded recently.

Table 1. BUSCO scores for the D. chrysippus genome assembly and each D. plexippus assembly in addition to the protein sequences resulting from both the original and re-annotation of each of these assemblies (using the insecta_odb10 BUSCO set, $n=1,367$ ).

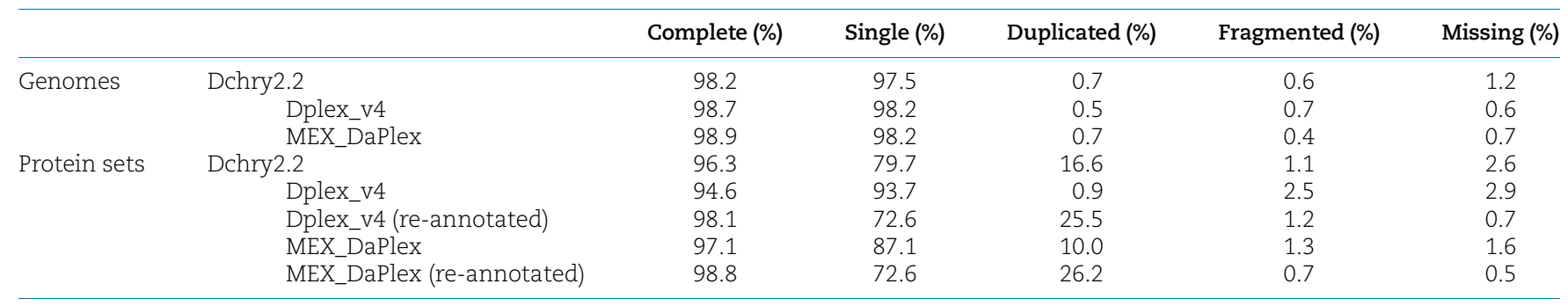

relative to D. plexippus. One of these, on chromosome 15, was identified previously as the site of a large expansion in gene copy number through multiple duplications and is associated with subspecies differentiation and color pattern variation in $D$. chrysippus (Martin et al. 2020). Further work to dissect this genomic region and compare chromosome structure among D. chrysippus subspecies is ongoing.

In total, the $354 \mathrm{Mb}$ D. chrysippus genome is $42-44 \%$ larger than that of D. plexippus (245-248 Mb). This difference is consistent for all autosomes, but most dramatic for the 3 chromosomes carrying large repetitive/duplicated tracts: namely 15, 26, and 29 (Fig. 2). By contrast, the $Z$ sex chromosome is nearly identical in size in the 2 species. This difference in autosome sizes could be explained either via a systematic size reduction in the lineage leading to D. plexippus, or a systematic increase in the lineage leading to $D$. chrysippus. These hypotheses can be distinguished by comparison with assemblies of other members of the genus or outgroups in the future.

\section{Transposable element and repeat content}

In total, the D. chrysippus genome comprises 35.5\% repeats, with the largest proportion of these being retroelements which make up $11.9 \%$ of the genome sequence (Fig. 3). Repeat masking of each of the D. plexippus assemblies revealed that a substantially lower proportion of the genomes of these close relatives comprise repeats, only between $11.2 \%$ and $14.3 \%$. Each of the main classified repeat families is more abundant in D. chrysippus compared to $D$. plexippus, with the largest difference between the species observed for the rolling-circle family which represents $1.8 \%$ and $2 \%$ of the D. plexippus genome sequence, compared to $7.6 \%$ of the D. chrysippus sequence. The repeat landscape of D. chrysippus (Fig. 4) highlights a number of expanding repeat families, most strikingly the rolling-circle repeats RC/Helitron (pink; Fig. 4). The increased prevalence of repetitive elements within the $D$. chrysippus genome (91-98 Mb more than D. plexippus) largely explains the larger genome size of D. chrysippus compared to D. plexippus (an increase of 106-109 Mb). Although the repeat content of genomes across the Lepidoptera order has been shown to vary substantially (Talla et al. 2017), our results suggest that even within a genus a large amount of variation can be present. Although the genome of $D$. chrysippus is rather repetitive, even within Lepidoptera (With repeats making up $35.5 \%$ of the genome), D. plexippus tends toward the lower end of repeat content (repeats make up 11.2$14.3 \%$ of the genome). 
(a) Exons

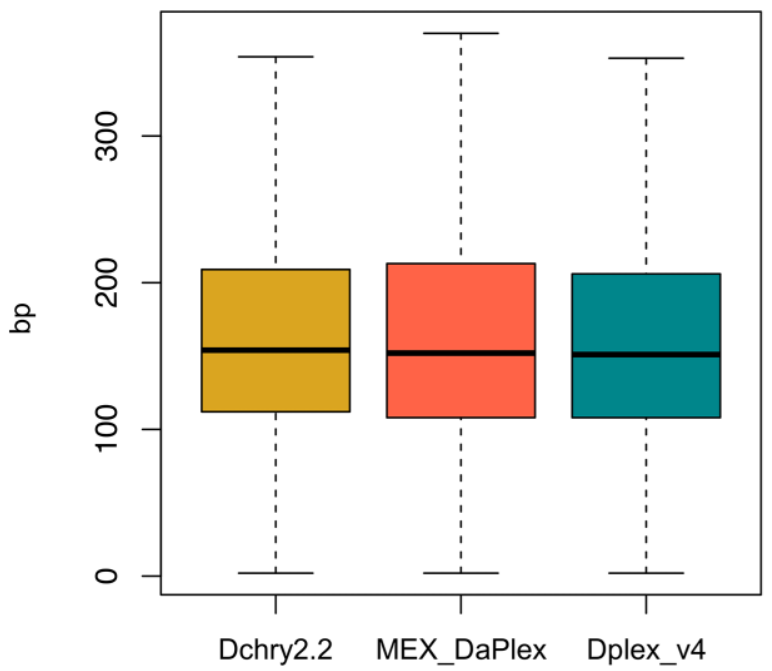

(b) Introns

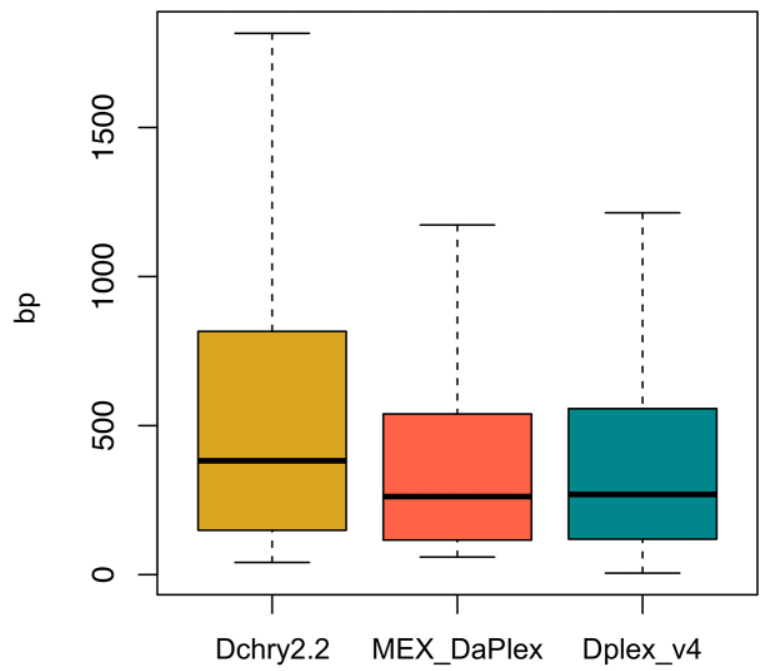

Fig. 5. Boxplots showing the distribution of (a) exon lengths and (b) intron lengths taken from the longest transcripts annotated with BRAKER2 for the 3 assemblies, D. chrysippus (Dchry2.2) and each of the re-annotated D. plexippus assemblies (MEX_DaPlex and Dplex_v4). Outlier points were omitted for clarity. Mean exon length is 226 bp in Dchry2.2, 238 bp for the re-annotated MEX_DaPlex assembly, and 217 bp for the re-annotated Dplex_v4 assembly. Mean intron length is $975 \mathrm{bp}$ in Dchry2.2, 665 bp for the re-annotated MEX_DaPlex assembly, and 738 bp for the re-annotated Dplex_v4 assembly.

\section{Gene content}

In total 16,260 protein-coding genes were annotated in the assembly by BRAKER2 (with 19,639 protein-coding mRNAs annotated-accounting for multiple transcripts/isoforms of the same gene), which included 136,694 exons and 117,106 introns. This number of genes is similar to that of the published annotations for each of the D. plexippus assemblies, which annotated 15,006 (Dplex_v4) and 15,995 (MEX_DaPlex) genes (as well as our reannotated versions of these assemblies which annotated 18,663 and 21,311 genes; in both cases, our annotation involved annotating additional smaller scaffolds not annotated in the original assemblies-284 vs. 30 scaffolds for Dplex_v4 and 64 vs. 55 for MEX_DaPlex). An analysis of BUSCOs using the insecta_odb10 benchmarking set shows that the full-genome sequences and annotated protein set for D. chrysippus are $98.2 \%$ and $96.3 \%$ complete for BUSCOs, respectively. This percentage completeness is close to that of both published D. plexippus annotations which have 94.6\% (Dplex_v4) and 97.1\% (MEX_DaPlex) complete BUSCOs (Table 1). Pannzer2 allowed us to add functional annotation to 9,567 of the full 16,260 gene set (functional annotation available at https://doi.org/10.5281/zenodo.5731560).

\section{Intron and exon length}

Exon length is relatively consistent across the 3 genomes, ranging from 217 bp (Dplex_v4 re-annotation) to 238 bp (MEX_DaPlex re-annotation; Fig. 5a). However, the mean intron length in the D. Chrysippus assembly ( $975 \mathrm{bp}$ ) is higher than that in the $2 \mathrm{D}$. plexippus assemblies (665 and 738 bp, respectively; Fig. 5b). This substantial increase in intron length in D. chrysippus likely explains the remaining variation in genome size between $D$. chrysippus and D. plexippus. This difference may represent a neutral increase in introns in D. chrysippus or a selection-mediated reduction in intron size in $D$. plexippus. These hypotheses may be resolved by comparison with genomes of other members of the genus in the future.

\section{Conclusions}

We have assembled a nearly chromosome-level genome for D. chrysippus, which is highly comparable in its quality to the best available assembly for D. plexippus. Although the 2 species retain strong synteny, the D. chrysippus genome is $>40 \%$ larger, with more repetitive content and larger introns on average. This implies stronger selection to limit nonessential DNA in D. plexippus. Future comparative studies involving other members of the genus could shed light on the processes and forces driving the evolution of genome size. The $D$. chrysippus genome will also serve as a reference for population genomic studies to test hypotheses about the evolution of warning coloration, host-parasite interactions, and the consequences of chromosomal rearrangements.

\section{Data availability}

The assembly and annotation are available at the European Nucleotide Archive project accession: PRJEB47812. Additional data files are provided at https://doi.org/10.5281/zenodo.5731560: purged haplotigs, assembly before manual edits, details of manual edits made to the assembly, and repeat library and functional annotation files. Scripts for genome assembly are available at https://github.com/kumarsaurabh20/DChry2.1 (last accessed 5 October 2021) and scripts for the genome annotation and analysis of introns and exons at https://github.com/RishiDeKayne/ Danaus_Dchry2.2_annotation (last accessed 5 October 2021).

Supplemental material is available at G3 online.

\section{Acknowledgments}

We thank Alexander Mackintosh for providing advice on genome assembly and annotation.

\section{Funding}

This work was supported by a Royal Society University Research Fellowship (URF\R1\180682) and Enhancement Award (RGF\EA 181071) awarded to SHM, a Swiss National Science Foundation Early Postdoc Mobility Fellowship (P2BEP3_195567) 
awarded to RD-K, and a European Research Council Horizon 2020 research and innovation program grant 646625 awarded to CB.

\section{Conflicts of interest}

None declared.

\section{Literature cited}

Ahola V, Lehtonen R, Somervuo P, Salmela L, Koskinen P, Rastas P, Välimäki N, Paulin L, Kvist J, Wahlberg N, et al. The Glanville fritillary genome retains an ancient karyotype and reveals selective chromosomal fusions in Lepidoptera. Nat Commun. 2014;5:4737.

Chakraborty M, Baldwin-Brown JG, Long AD, Emerson JJ. Contiguous and accurate de novo assembly of metazoan genomes with modest long read coverage. Nucleic Acids Res. 2016;44(19):e147.

Chin C-S, Peluso P, Sedlazeck FJ, Nattestad M, Concepcion GT, Clum A, Dunn C, O'Malley R, Figueroa-Balderas R, Morales-Cruz A, et al. Phased diploid genome assembly with single-molecule real-time sequencing. Nat Methods. 2016;13(12):1050-1054.

Cicconardi F, Lewis JJ, Martin SH, Reed RD, Danko CG, Montgomery $\mathrm{SH}$. Chromosome fusion affects genetic diversity and evolutionary turnover of functional loci, but consistently depends on chromosome size. Mol Biol Evol. 2021;38(10):4449-4462.

Gremme G, Steinbiss S, Kurtz S. GenomeTools: a comprehensive software library for efficient processing of structured genome annotations. IEEE/ACM Trans Comput Biol Bioinform. 2013;10(3): 645-656.

Gu L, Reilly PF, Lewis JJ, Reed RD, Andolfatto P, Walters JR. Dichotomy of dosage compensation along the neo $\mathrm{Z}$ chromosome of the monarch butterfly. Curr Biol. 2019;29(23):4071-4077.

Brina T, Hoff KJ, Lomsadze A, Stanke M, Borodovsky M. BRAKER2: Automatic eukaryotic genome annotation with GeneMark-EP+ and AUGUSTUS supported by a protein database. NAR genomics and bioinformatics, 2021;3(1):lqaa108.

Kim KW, De-Kayne R, Gordon IJ, Omufwoko KS, Martins DJ, FfrenchConstant R, Martin SH. Stepwise evolution of a butterfly supergene via duplication and inversion. bioRxiv 2021.12.06.471392; doi: https://doi.org/10.1101/2021.12.06.471392.

Koren S, Walenz BP, Berlin K, Miller JR, Bergman NH, Phillippy AM. Canu: scalable and accurate long-read assembly via adaptive $\mathrm{k}$ mer weighting and repeat separation. Genome Res. 2017;27(5): 722-736.

Krueger F. 2012. Trim Galore: a wrapper tool around Cutadapt and FastQC to consistently apply quality and adapter trimming to FastQ files, with some extra functionality for MspI-digested RRBS-type (Reduced Representation Bisufite-Seq) libraries. http://www bioinformatics babraham ac uk/projects/trim_ga lore/ [accessed 1 July 2018].

Lewis JJ, Cicconardi F, Martin SH, Reed RD, Danko CG, et al. The Dryas iulia genome supports multiple gains of a $\mathrm{W}$ chromosome from a B chromosome in butterflies. Genome Biol. Evol. 2021;13:evab128.

Li H. Minimap2: pairwise alignment for nucleotide sequences. Bioinformatics. 2018;34(18):3094-3100.

Li H, Durbin R. Fast and accurate long-read alignment with BurrowsWheeler transform. Bioinformatics. 2010;26(5):589-595.

Li H, Handsaker B, Wysoker A, Fennell T, Ruan J, Homer N, Marth G, Abecasis G, Durbin R; 1000 Genome Project Data Processing Subgroup. The Sequence Alignment/Map format and SAMtools. Bioinformatics. 2009;25(16):2078-2079.

Lushai G, Smith DAS, Gordon IJ, Goulson D, Allen JA, Maclean N. Incomplete sexual isolation in sympatry between subspecies of the butterfly Danaus chrysippus (L.) and the creation of a hybrid zone. Heredity (Edinb). 2003;90(3):236-246.

Marçais G, Delcher AL, Phillippy AM, Coston R, Salzberg SL, Zimin A. MUMmer4: a fast and versatile genome alignment system. PLoS Comput Biol. 2018;14(1):e1005944.

Martin SH, Singh KS, Gordon IJ, Omufwoko KS, Collins S, Warren IA, Munby H, Brattström O, Traut W, Martins DJ, et al. Whole-chromosome hitchhiking driven by a male-killing endosymbiont. PLoS Biol. 2020;18(2):e3000610.

Mongue AJ, Nguyen P, Voleníková A, Walters JR. Neo-sex chromosomes in the monarch butterfly, Danaus plexippus. G3 (Bethesda). 2017;7(10):3281-3294.

Ranz JM, González PM, Clifton BD, Nazario-Yepiz NO, HernándezCervantes PL, Palma-Martínez MJ, Valdivia DI, Jiménez-Kaufman A, Lu MM, Markow TA, et al. A de novo transcriptional atlas in Danaus plexippus reveals variability in dosage compensation across tissues. Commun Biol. 2021;4(1):791.

Rhie A, Walenz BP, Koren S, Phillippy AM. Merqury: reference-free quality, completeness, and phasing assessment for genome assemblies. Genome Biol. 2020;21(1):27.

Roach MJ, Schmidt SA, Borneman AR. Purge Haplotigs: allelic contig reassignment for third-gen diploid genome assemblies. BMC Bioinformatics. 2018;19(1):460.

Simão FA, Waterhouse RM, Ioannidis P, Kriventseva EV, Zdobnov EM. BUSCO: assessing genome assembly and annotation completeness with single-copy orthologs. Bioinformatics. 2015;31(19): 3210-3212.

Smit AFA, Hubley R. RepeatModeler Open-1.0. 2008. http://www repeatmasker org [accessed 2021 August 20].

Smit AFA, Hubley R, Green P. RepeatMasker Open-4.0. 2015. http: //www.repeatmasker.org [accessed 2021 August 20].

Smith DAS, Gordon IJ, Traut W, Herren J, Collins S, Martins DJ, Saitoti K, Ireri P, Ffrench-Constant R. A neo-W chromosome in a tropical butterfly links colour pattern, male-killing, and speciation. Proc R Soc B. 2016;283(1835):20160821.

Smith DAS, Owen DF, Gordon IJ, Lowis NK. The butterfly Danaus chrysippus (L.) in East Africa: polymorphism and morph-ratio clines within a complex, extensive and dynamic hybrid zone. Zool J Linn Soc. 1997;120(1):51-78.

Talla V, Suh A, Kalsoom F, Dinca V, Vila R, Friberg M, Wiklund C, Backström N. Rapid increase in genome size as a consequence of transposable element hyperactivity in wood-white (Leptidea) butterflies. Genome Biol Evol. 2017;9(10):2491-2505.

Tan W-H, Acevedo T, Harris EV, Alcaide TY, Walters JR, Hunter MD, Gerardo NM, de Roode JC. Transcriptomics of monarch butterflies (Danaus plexippus) reveals that toxic host plants alter expression of detoxification genes and down-regulate a small number of immune genes. Mol Ecol. 2019;28(22):4845-4863.

Törönen P, Medlar A, Holm L. PANNZER2: a rapid functional annotation web server. Nucleic Acids Res. 2018;46(W1):W84-W88.

Vaser R, Sović I, Nagarajan N, Šikić M. Fast and accurate de novo genome assembly from long uncorrected reads. Genome Res. 2017; 27(5):737-746.

Walker BJ, Abeel T, Shea T, Priest M, Abouelliel A, Sakthikumar S, Cuomo CA, Zeng Q, Wortman J, Young SK, et al. Pilon: an integrated tool for comprehensive microbial variant detection and genome assembly improvement. PLoS One. 2014;9(11):e112963.

Zhan S, Zhang W, Niitepõld K, Hsu J, Haeger JF, Zalucki MP, Altizer S, de Roode JC, Reppert SM, Kronforst MR, et al. The genetics of monarch butterfly migration and warning colouration. Nature. 2014; 514(7522):317-321. 